\title{
Expression of the neuron-specific protein CHD5 is an independent marker of outcome in neuroblastoma
}

Idoia Garcia', Gemma Mayol', Eva Rodríguez', Mariona Suñol², Timothy R Gershon³, José Ríos, Nai-Kong V Cheung ${ }^{5}$, Mark W Kierann ${ }^{6}$, Rani E George ${ }^{6}$, Antonio R Perez-Atayde, Carla Casala ${ }^{1}$, Patricia Galván ${ }^{1}$, Carmen de Torres $^{1}$, Jaume Mora ${ }^{1 *}$, Cinzia Lavarino ${ }^{1 *}$

\begin{abstract}
Background: The chromodomain, helicase DNA-binding protein 5 (CHD5) is a potential tumor suppressor gene located on chromosome 1p36, a region recurrently deleted in high risk neuroblastoma (NB). Previous data have shown that CHD5 mRNA is present in normal neural tissues and in low risk NB, nevertheless, the distribution of CHD5 protein has not been explored. The aim of this study was to investigate CHD5 protein expression as an immunohistochemical marker of outcome in NB. With this purpose, CHD5 protein expression was analyzed in normal neural tissues and neuroblastic tumors (NTs). CHD5 gene and protein expression was reexamined after induction chemotherapy in a subset of high risk tumors to identify potential changes reflecting tumor response.

Results: We provide evidence that CHD5 is a neuron-specific protein, absent in glial cells, with diverse expression amongst neuron types. Within NTs, CHD5 immunoreactivity was found restricted to differentiating neuroblasts and ganglion-like cells, and absent in undifferentiated neuroblasts and stromal Schwann cells. Correlation between protein and mRNA levels was found, suggesting transcriptional regulation of CHD5. An immunohistochemical analysis of 90 primary NTs highlighted a strong association of CHD5 expression with favorable prognostic variables (age at diagnosis $<12$ months, low clinical stage, and favorable histology; $\mathrm{P}<0.001$ for all), overall survival (OS) $(P<0.001)$ and event-free survival $(E F S)(P<0.001)$. Multivariate analysis showed that CHD5 prognostic value is independent of other clinical and biologically relevant parameters, and could therefore represent a marker of outcome in NB that can be tested by conventional immunohistochemistry. The prognostic value of CHD5 was confirmed in an independent, blinded set of 32 NB tumors $(P<0.001)$.

Reactivation of CHD5 expression after induction chemotherapy was observed mainly in those high risk tumors with induced tumor cell differentiation features. Remarkably, these NB tumors showed good clinical response and prolonged patient survival.
\end{abstract}

Conclusions: The neuron-specific protein CHD5 may represent a marker of outcome in NB that can be tested by conventional immunohistochemistry. Re-establishment of CHD5 expression induced by chemotherapy could be a surrogate marker of treatment response.

\footnotetext{
* Correspondence: jmora@hsjdbcn.org; clavarino@fsjd.org

'Developmental Tumor Biology Laboratory, Hospital Sant Joan de Déu,

Fundación Sant Joan de Déu, Barcelona, Spain

Full list of author information is available at the end of the article
} 


\section{Introduction}

Neuroblastic tumors (NTs) are embryonal cancers arising from neural crest derived sympathetic nervous system precursors. These neoplasms are the most common extracranial solid tumors in childhood and account for approximately $15 \%$ of all pediatric oncology deaths [1].

Neuroblastoma (NB), the most undifferentiated form of NTs, embodies a heterogeneous spectrum of diseases whereby patients with similar clinicopathological features exhibit radically different outcomes ranging from spontaneous regression to inexorable progression. Since treatment strategies vary from a "watchful waiting" approach to multimodal intensive regimens, precise risk assessment is critical for therapeutic decisions. Various combinations of prognostic markers have been used with success for risk group distinction, including clinical, histologic and genetic factors, yet there remain cases where established indicators of aggressiveness have demonstrated limited clinical utility. Additional parameters are therefore needed for a more precise identification and therapeutic targeting of high risk NB patients.

There is an apparent link between NB aggressiveness and specific genetic aberrations. One of the most recurrent genetic alterations described is the deletion of the short arm of chromosome 1 found in approximately $35 \%$ of NB [2]. The high incidence of chromosome $1 \mathrm{p}$ deletion in human cancer [2], with $1 \mathrm{p} 36$ deletion being the most common alteration [3], has led to an extensive search for 1 p36 tumor suppressor genes. Recent findings have identified the CHD5 gene as a candidate tumor suppressor [4,5] mapping to the smallest region of deletion (SRD) described in NB, 1p36.31 [6]. Evidence supporting CHD5 as a tumor suppressor is the recently reported strong promoter methylation and transcriptional silencing of the remaining allele in $1 \mathrm{p}$ deleted $\mathrm{NB}$ cell lines [5]. Nevertheless, low or absent CHD5 expression levels have been found in NB cell lines lacking promoter methylation [7], $1 \mathrm{p}$ deletion, or inactivating mutations [6], suggesting other mechanisms by which CHD5 expression may be inhibited.

CHD5 is one of the nine members of the chromodomain helicase DNA-binding (CHD) family of enzymes that belong to the ATP-dependent chromatin remodeling protein SNF2 superfamily [8]. CHD protein structure is characterized by two $\mathrm{N}$-terminal chromodomains and a SNF2-like ATPase central domain that defines the chromodomain remodeling proteins $[9,10]$. The members of this evolutionarily conserved class of proteins play a critical role in organizing the chromatin structure and accordingly, in chromatin based transcriptional regulation of genes.
The aberrant expression of some of the CHD genes has been associated with human disease processes like CHARGE syndrome, Hodgkin's lymphoma or dermatomyositis [8]. CHD5 mRNA expression, restricted to neuronal-derived tissues and the adrenal gland in normal tissues [10], is basically absent in NB primary tumors with high risk features, $M Y C N$ amplification, advanced stage and 1p monosomy [5].

The distribution of CHD5 protein in NTs and normal neural tissues has not been explored. Like neural tissue, NTs consist of two main cell populations, neuroblastic cells and Schwann-like cells. The malignant potential of these tumors is inherently dependent on the proportion of immature neuroblastic cells and the abundance of Schwann cell stromal component, Schwannian stromapoor undifferentiated NB being the most malignant. CHD5 expression remains to be investigated in these two cell populations. In the present study, we analyzed by immunohistochemistry normal neural derived tissues and NTs to visualize CHD5 protein distribution within the different cell populations. Because impaired $\mathrm{CHD} 5$ expression is associated with high risk NB tumors, we asked whether CHD5 protein expression might serve as an immunohistochemical marker of outcome in NB. It is known that gene expression pattern can change with treatment, for this reason, CHD5 gene and protein expression was re-examined after induction treatment in a set of paired cases.

\section{Material and Methods}

Patients and tumor samples

A total of 90 primary tumor specimens (63 NB, 14 ganglioneuroblastomas (GNB) and 13 ganglioneuromas $(\mathrm{GN})$ ) (Additional file 1) were obtained at diagnosis from two institutions (Hospital Sant Joan de Déu (HSJD) of Barcelona and Memorial Sloan-Kettering Cancer Center (MSKCC) of New York) together with 12 high risk NB cases with available paired diagnostic and post-chemotherapy tumor specimens. An independent set of 32 NB tumors was obtained from Children's Hospital of Boston and Dana-Farber Cancer Institute (CHB/ DFCI) for data validation analysis. Non-tumor samples (fetal brain, adult cerebral cortex, adult cerebellum, adrenal gland, bone marrow, spinal cord and sympathetic ganglion) were also included in this study.

NB risk assessment was defined by the International Neuroblastoma Staging System (INSS) [11]. NB stages 1, 2, 3 (MYCN non-amplified) and 4s were uniformly treated without use of cytotoxic therapy, when possible. Stage 4 and stage $3 M Y C N$ amplified NB patients were treated according to N5, N6 or N7 protocols. This study was approved by the Institutional Review Boards and informed consent was obtained before collection of samples. 
Tumors were assessed by a pathologist (M.S.), only tumors with $>70 \%$ viable tumor cell content were included in the study.

Seven NB cell lines (LA-N-1, SKNSH-SY5Y, SK-N-Be (2)C, SKNSH-EP1, SK-N-JD, SK-N-LP and SK-N-AS) were used in this study. NB cell lines were cultured in RPMI-1640 supplemented with $10 \%$ fetal bovine serum (FBS), $2 \mathrm{mM}$ L-glutamine and penicillin $(100 \mathrm{U} / \mathrm{ml})$ and streptomycin $(100 \mu \mathrm{g} / \mathrm{ml})$ (GIBCO, Invitrogen, US) at $37^{\circ} \mathrm{C}$ in $5 \% \mathrm{CO}_{2}$ atmosphere.

\section{In vivo study}

NB cell lines SK-N-JD, SK-N-LP and SK-N-AS were harvested and resuspended in phosphate buffered saline (PBS) solution and BD Matrigel Basement Membrane Matrix (BD Biosciences, US). One hundred microliters of cell suspension containing $8 \times 10^{6}$ cells were subcutaneously inoculated into the right flank of six-week old CD-1 Nude (nu/nu) mice (Charles River Laboratories, Europe). Mice were killed when NB cell lines developed tumors that exceeded $1.5 \mathrm{~cm}^{3}$. Tumors were removed surgically, fixed in $10 \%$ formalin and embedded in paraffin for histological examination.

\section{Immunohistochemistry}

Immunohistochemical (IHC) analysis was performed on formalin-fixed, paraffin-embedded (FFPE) tissues using rabbit-polyclonal anti-CHD5 antibody (Strategic Diagnostics, DE) at a 1:1000 dilution for 1 hour; mouse-polyclonal anti-Neurofilament protein, 68kD (NF68) antibody (Zymed, US) 1:300 dilution, 1 hour and mouse-polyclonal anti-Glial fibrillary acidic protein (GFAP) antibody (Novocastra, UK) 1:200 dilution, 2 min. Two different anti-CHD5 antibody batches (T00251-A1 and T00251-A02, Strategic Diagnostics, DE) have been tested in this study. Normal human brain was used as positive control.

Slides were examined by a pathologist (M.S.) using an Olympus BX41 light microscopy to assess staining and score both percentage of positive cells and staining intensity ( 0 , negative; 1 , weak; 2 , strong and 3 , very intense staining). Integer values were assigned to the proportion of positive cells $(<25 \%=1 ; 25-75=2 ;>75 \%$ $=3$ ). Intensity and positive cell values were multiplied to provide a single score for each case.

Double fluorescent immunostaining: Paraformaldehyde (4\%, pH 7.4) fixed cryosections, blocked with bovine serum albumin (BSA) 1\% for 1 hour, were incubated overnight at $4^{\circ} \mathrm{C}$ with a rabbit-polyclonal anti-CHD5 antibody (H-185) (Santa Cruz, US) at 1:1000 dilution, followed by anti-rabbit IgG Cy3-conjugated antibody, (Sigma, US) 1:400 dilution for $45 \mathrm{~min}$. Sections were subsequently incubated with anti-NF68 antibody (1:300 dilution) 1 hour or anti-GFAP antibody (1:200 dilution)
$2 \mathrm{~min}$, and stained with anti-mouse IgG FITC-conjugated antibody (Sigma, US) 1:700 dilution, $45 \mathrm{~min}$. Nuclei were counterstained with 4'6-diamino-2-phenylindole (DAPI) (Sigma, US), 1:5000 dilution, $5 \mathrm{~min}$.

Paraformaldehyde fixed bone marrow aggregates were incubated with anti-GD2 antibody (BD Biosciences, US) 1:800 dilution 1 hour and stained with anti-mouse IgGFITC antibody at 1:700 dilution, $45 \mathrm{~min}$, or with antiCHD5 antibody as described above.

Immunoreactivity was evaluated with a Leica epifluorescence DM5000B microscope (Leica Microsystems, US).

\section{Western blot analysis}

Proteins were extracted from cell lines and homogenized tissue in lysis buffer (20 mM Tris $\mathrm{pH} 8.8,80 \mathrm{mM} \mathrm{NaCl}$, $1 \%$ NP-40 and protease inhibitors). Protein concentrations were quantified using the Bradford method (BioRad laboratories, US) and $30 \mu \mathrm{g}$ of protein were resolved on an 8\% SDS-PAGE. Membranes were incubated with polyclonal anti-CHD5 antibody (1:2000; Strategic Diagnostics, DE) and monoclonal anti $\beta$-actin antibody (1:5000; Sigma, US) and detected with donkey anti-rabbit IgG HRP-conjugated antibody (1:2500; Affinity BioReagents, Inc., US) and goat anti-mouse IgG HRPconjugated antibody (1:5000; Sigma, US) respectively. Antibody conjugates were visualized by enhanced chemiluminescence (ECL, Amersham Life Science, US).

\section{RNA isolation and CDNA synthesis}

Total RNA was isolated from snap frozen samples and cell lines using Tri Reagent (Sigma, US), following manufacturers' protocols. cDNA was synthesized from $1 \mu \mathrm{g}$ total RNA using random primers and M-MLV reverse transcriptase (Promega, US) as previously described [12].

\section{Quantitative Real-time Polymerase Chain Reaction (qRT- PCR)}

Quantification of transcript levels, using the $\Delta \Delta C_{T}$ relative quantification method, were performed on an $\mathrm{ABI}$ Prism 7000 Sequence Detection System with TaqMan ${ }^{\circ}$ Assay-on-Demand Gene Expression products (Applied Biosystems, US), as previously reported [12].

\section{Statistical analysis}

Comparisons between immunohistochemical results were performed by means of the log-rank test. qRTPCR transcript levels were normalized by z-score transformation to enable a correlation analysis with the immunostaining score values. Correspondence between immunoreactivity and mRNA expression levels within the same samples was examined using the Spearman's correlation coefficient analysis. Statistical analyses for qualitative variables were performed by means of the 
Fisher's exact test and U Mann-Whitney test for quantitative or ordinal variables. Overall survival (OS) and event-free survival (EFS) probabilities were estimated using the Kaplan-Meier method. Multivariate Cox regression models were used to examine the prognostic significance of CHD5, INSS stage, age at diagnosis, MYCN status and 1p LOH. Each variable consisted of two groups: "INSS stage" consisted of: (1) ST1, 2, 3 and 4s, and (2) ST4; "age" (at diagnosis): (1) $\leq 12$ months (2) > 12 months; "MYCN": (1) MYCN non-amplified (2) MYCN amplified; "LOH": (1) no LOH (2) LOH. Predictive Positive and Negative Values (PPV and NPV) were used for a descriptive comparison between CHD5 expression and $M Y C N$ and $1 \mathrm{p} \mathrm{LOH}$. All reported $\mathrm{P}$-values are two-sided. P-values $\leq 0.05$ were considered statistically significant. Statistical analysis was performed with SPSS 15.0 package (SPSS, Chicago, IL).

\section{Results \\ CHD5 protein expression in normal neural tissues is restricted to neuronal cells}

In normal human neural tissue sections (brain cortex, cerebellum, spinal cord and sympathetic ganglion), CHD5 immunoreactivity was found restricted to neurons, whereas glial cells were consistently negative (Figure $1 \mathrm{~A}, \mathrm{C}, \mathrm{D}$ and $1 \mathrm{E})$. CHD5 expression pattern was confirmed by immunostaining with neuronal (NF68) and glial (GFAP) cell markers. Frozen brain sections analyzed by double immunofluorescence showed colocalization of CHD5 and NF68 in neurons. No CHD5 protein expression was observed in GFAP positive glial cells (Figure $1 \mathrm{G}$ and $1 \mathrm{H}$ ).

Intensity and intracellular localization of CHD5 staining in the cerebral cortex varied among neuron types but did not exhibit a layer-related expression (Figure 1A). Nuclear labeling was intense in morphologically small neurons with scarce cytoplasm present in all cortical layers identified by size and location as interneurons. Larger neurons with triangular shaped soma, including pyramidal neurons present in cortical layers III, IV and $\mathrm{V}$, exhibited essentially negative or lower intensity of nuclear staining and diffuse cytoplasm reactivity (Figure 1A). In the cerebellum, Purkinje cells and deep nuclei neurons exhibited intense nuclear and diffuse cytoplasm staining. Cerebellar granular layer neurons lacked immunoreactivity (Figure 1C).

Spinal cord specimens were characterized by intense positive neuron processes, predominantly located in the external white matter, and large motoneuron cell bodies with positive cytoplasm and mostly negative nuclear staining (Figure 1D). All glial cells, including the ependymal cells lining the central canal of the spinal cord, were negative for CHD5 expression (Figure 1D*). In the sympathetic ganglia, neuron cell bodies showed intense nuclear and diffuse cytoplasm reactivity, while the stromal cell component was found negative for CHD5 (Figure $1 \mathrm{E})$.

Adrenal gland specimens exhibited weak CHD5 expression, mainly in the nucleus of the medullary cells. Neuroblastic aggregates found in fetal adrenal glands (19-20 weeks) were essentially negative, although few intermixed positive cells were identified in larger neuroblastic islets (Figure 1F).

CHD5 expression was evaluated in brain cortex specimens and in NB cell lines by immunoblot analysis. CHD5 protein (250-260 kDa) was detected only in brain cortex specimens, both in the total protein extract and in the nuclear fraction. No CHD5 protein was detected in the cytoplasmic fraction of all the analyzed specimens or in NB cell lines (Figure 1B).

These results identify CHD5 as a neuron-specific protein, absent in glial cells, with a diverse expression pattern amongst neuron types. Human immature neuroblastic aggregates in the developing adrenal gland are mostly negative for CHD5.

\section{CHD5 protein is expressed in the neuroblastic component of low clinical risk NTs}

CHD5 immuno-localization was investigated in a total of 90 primary NTs (63 NB including 24 stage 4, 8 stage $4 \mathrm{~s}$ and 31 loco-regional NB; $14 \mathrm{GNB}$ and $13 \mathrm{GN}$ ) (Table 1).

Stage 4 NB cases, all histologically undifferentiated high risk NB, appeared predominantly (20/24) negative or with $<25 \%$ neuroblastic cells with faint CHD5 nuclear reactivity (Figure $2 \mathrm{~A}$ and $2 \mathrm{E}$; Table 1 , Additional file 1). Only $3 / 24$ undifferentiated NB tumors exhibited weak nuclear reactivity in $25-75 \%$ of cells, and one had intense nuclear staining in $>75 \%$ of tumor cells. In contrast, stage $4 \mathrm{~s} \mathrm{NB}$, histologically undifferentiated low risk tumors, showed consistently (8/8) very intense CHD5 nuclear positivity in $>75 \%$, generally $>90 \%$ of the neuroblasts (Figure 2F, Table 1, Additional file 1). This clinically low risk NB is, nevertheless, a highly proliferative metastatic tumor. Thus, for 2 stage $4 \mathrm{~s}$ NB tumors, CHD5 expression was also evaluated in the liver and bone marrow metastases. Intense CHD5 immunopositivity, equivalent to the primary tumor, was observed in $>75 \%$ neuroblasts disseminated in the liver. Intriguingly, bone marrow neuroblastic aggregates, identified using an antibody against the ganglioside GD2 ubiquitously expressed in NB (data not shown), lacked CHD5 immunoreactivity (Figure $2 \mathrm{H}$ ), similar to stage 4 bone marrow smears (Figure 2G).

Loco-regional tumors (stage 1, 2, and 3) displayed more heterogeneous expression patterns (Figure $2 \mathrm{~B}$ and $2 \mathrm{C}$; Additional file 1), with staining values being highest in differentiating $\mathrm{NB}$, where intense nuclear staining was 

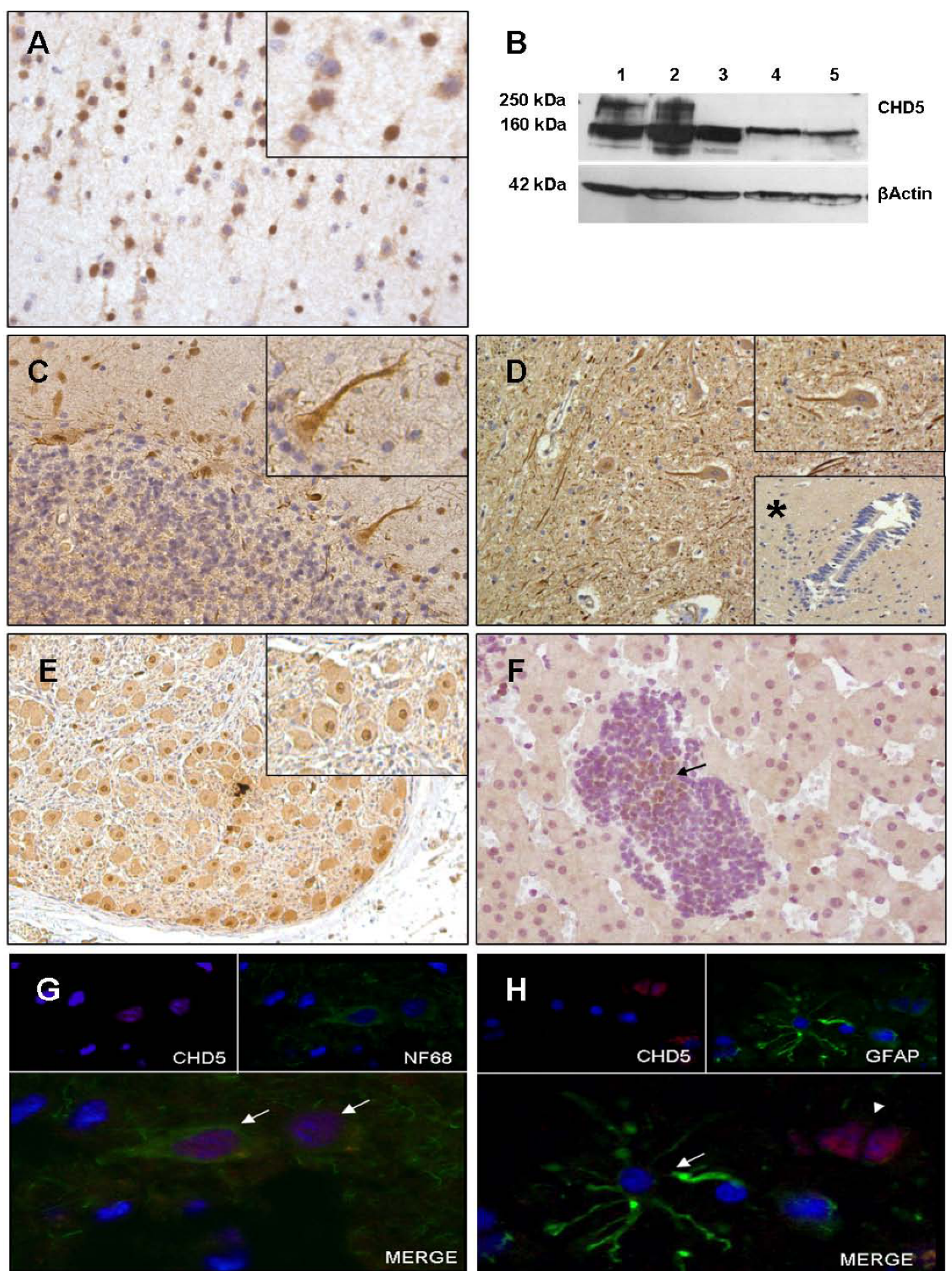

Figure 1 CHD5 protein expression in normal human neural tissues. CHD5 immunostaining in (A) normal neural tissue, cerebral cortex (100x), box: pyramidal and interneuron CHD5 staining (400x); (B) expression of CHD5 protein detected by immunoblotting in (1) brain cortex total protein, (2) brain cortex nuclear protein fraction, (3) brain cortex cytoplasmatic fraction, (4) LA-N-1 NB cell line total protein and (5) LA-N-1 nuclear fraction; CHD5 predicted molecular weight of 250-260 kDa is based on the amino acid composition (ref. 5); all analyzed samples displayed a 150-160 kDa size band, not yet characterized; (C) Cerebellum (100x), box: Purkinje cell CHD5 staining (400x); (D) Spinal cord (100x), box: Motoneuron CHD5 staining (400x), box*: ependymal cells lining canal spinal cord (100x); (E) Sympathetic ganglia (100x), box: Ganglion cell CHD5 staining (400x); (F) immature neuroblast aggregates within fetal adrenal gland (200x), (arrow) CHD5 immunopositive neuroblasts; (G-H) double fluorescent immunostaining, (G, arrow) NF68 ${ }^{+} / \mathrm{CHD5}^{+}$neuron; ( $\mathrm{H}$, arrow) GFAP ${ }^{+} / \mathrm{CHD} 5^{-}$astrocyte, $\left(\mathrm{H}\right.$, arrow head) $\mathrm{GFAP}^{-} / \mathrm{CHD}^{+}$neuron. 
Table 1 CHD5 inmunostaining in Neuroblastic tumors

\begin{tabular}{ccccc}
\hline & \multicolumn{4}{c}{ Percentage of CHD5 $\begin{array}{c}\text { immunopositive neuroblastic } \\
\text { cells }\end{array}$} \\
\cline { 3 - 5 } & $\mathbf{n}$ & $<\mathbf{2 5 \%}$ & $\mathbf{2 5 - 7 5 \%}$ & $>\mathbf{7 5 \%}$ \\
\hline St 1,2,3 & 31 & $10 / 31(32.2 \%)$ & $9 / 31(29 \%)$ & $12 / 31(38.7 \%)$ \\
St 4 & 24 & $19 / 24(79.1 \%)$ & $4 / 24(16.6 \%)$ & $1 / 24(4.1 \%)$ \\
St 4s & 8 & - & - & $8 / 8(100 \%)$ \\
GNB & 14 & $14 / 14^{*}(100 \%)$ & - & $14 / 14^{* *}(100 \%)$ \\
GN & 13 & - & - & $13 / 13(100 \%)$ \\
\hline Total & 90 & 29 & 13 & 48
\end{tabular}

Percentage of CHD5 positive (nuclear staining) tumor cells within each neuroblastic tumor group evaluated using predetermined cutoff values $(<25 \%$; $25-75 \% ;>75 \%)$. Detailed data regarding percentage of positive tumor cells and staining intensity are reported in Additional file 1. For GNB tumors, the undifferentiated neuroblastic $(*)$ and the ganglionar $\left({ }^{*}\right)$ cell populations were scored separately. GNB = ganglioneuroblastoma, GN = Ganglioneuroma.

observed in $>75 \%$ of neuroblastic cells (13/32) (Figure 2B; Additional file 1), and lowest in stage $3 M Y C N$ amplified NB composed mainly of undifferentiated neuroblasts with undetectable immunoreactivity, similar to stage 4 NB cases (Figure 2C, Table 1, Additional file 1).

GNB (14/14) and GN (13/13) tumors exhibited ganglion-like cells with intense nuclear and diffuse cytoplasm staining. Absence of nuclear staining and feeble cytoplasmic reactivity was observed in Schwann-like cells (Figure 2D; Additional file 1). The undifferentiated neuroblastic component of GNB lacked CHD5 staining (Table 1, Additional file 1).

The described immunohistochemical assays were performed using two different batches of the anti-CHD5 antibody (T00251-A1 and T00251-A02). Both batches performed consistently across many repeats, further supporting the validity of our results (Additional file 2A). The specificity of the anti-CHD5 antibody was further validated on mouse xenografts of human NB cell lines (SK-N-JD, SK-N-LP and SK-N-AS). All the xenografts were found to be negative for CHD5 staining (Additional file $2 \mathrm{~B}$ ).

Altogether, CHD5 protein was expressed in the nucleus of neuroblastic cells of clinical low risk NTs. In stage 4s NB, CHD5 negative neuroblast bone marrow metastasis imply the existence of intratumoral clones with CHD5 differential expression in an otherwise histologically homogeneous tumor subtype.

\section{CHD5 transcript levels are associated with protein expression}

CHD5 protein expression was contrasted with gene transcript levels. Quantification of CHD5 mRNA in non-tumoral frozen tissue samples using qRT-PCR identified high expression in fetal brain and adult cerebral cortex, as reported previously [10]. Normal bone marrow specimens lacked $C H D 5$ expression.
CHD5 mRNA levels were analyzed for 84 primary NTs obtained at diagnosis (23 stage $4 ; 7$ stage 4 s; 34 loco-regional NB; $9 \mathrm{GNB}$ and $11 \mathrm{GN})$; 55 of these tumors were also analyzed by immunohistochemistry.

High risk undifferentiated NB tumors, stage 4 and stage $3 M Y C N$-amplified NB displayed significantly lower mRNA expression levels than stage $1,2,3$ ( $\mathrm{P}<$ $0.001)$ and stage $4 \mathrm{~s} \mathrm{NB}(\mathrm{P}=0.001)$ (Additional file 3$)$. The highest mean expression values, similar to normal fetal brain, were found for stage $4 \mathrm{~s}$ NB. GN specimens displayed consistently low CHD5 transcript levels, whereas, GNB tumors were characterized by highly variable expression attributable to the presence of $\mathrm{CHD} 5$ negative component, Schwann-like stroma and undifferentiated neuroblasts, besides the positive ganglion-like cells that compose these tumors.

Correlation between CHD5 immunoreactivity and mRNA expression levels within the same samples was examined in a set of 34 consecutive NB tumors. Immunohistochemical and qRT-PCR analyses were carried out on the same portion of the tumor specimen, with similar cell composition and a high tumor cell content (>70\% as recommended for PCR studies). CHD5 nuclear immunoreactivity was assigned a staining score (Additional file 1) and gene expression values were $\mathrm{z}$-score transformed. A significant correlation was observed between mRNA and protein levels (Spearman's rho = 0.774; $\mathrm{P}<0.001$ ), low CHD5 protein scores were consistently associated with low mRNA levels (negative zscore values), and high IHC scores with high mRNA expression (positive z-score values) (Additional file 4). Interestingly, very intense nuclear staining displayed by low risk tumors, mostly stage $4 \mathrm{~s}$ and infant stage $1 \mathrm{NB}$, was not associated with the highest transcript levels (Additional file 4, cases \# 1-6, 30, 31 and 33).

These results reveal a correspondence between CHD5 protein and mRNA expression, suggesting a potential regulation of $C H D 5$ expression at the transcriptional level.

\section{CHD5 protein expression is associated with patient outcome in NB}

CHD5 nuclear immunoreactivity was assigned a staining score (Additional file 1) and compared to clinical and biological variables currently used for NB risk classification. High CHD5 staining values were found to be significantly associated with INSS stages 1, 2, 3 (MYCN non-amplified) and 4s NB ( $\mathrm{n}=63)$, age at diagnosis $<12$ $\mathrm{m}(\mathrm{n}=63)$ and favorable tumor histology $(\mathrm{n}=63) ; \mathrm{P}<$ 0.001 for all the tested variables.

To assess whether CHD5 expression was associated with patient outcome, immunoreactivity scores were compared to overall survival (OS) and event-free survival (EFS) for all 63 NB tumors. The median score value 


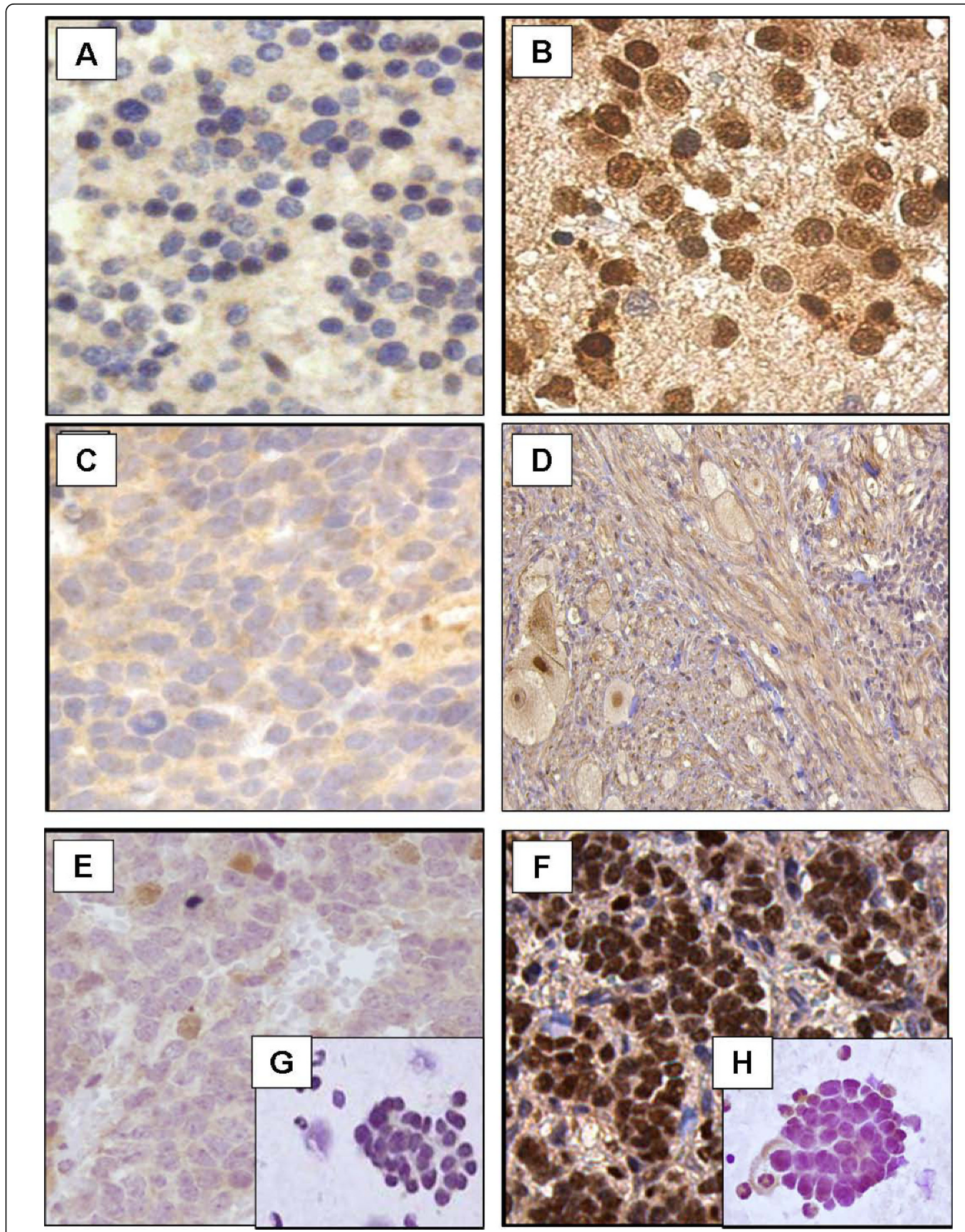

Figure 2 CHD5 protein expression in neuroblastic tumors. CHD5 immunostaining in (A) stage 4 undifferentiated NB (400x); (B) loco-regional differentiating NB (400x); (C) stage 3, MYCN amplified, undifferentiated NB (400x); (D) Ganglioneuroblastoma (100x); (E) stage 4 (200x) and (F) stage 4 s primary tumor (200x) with bone marrow neuroblast aggregates (G-H), respectively (400x). 


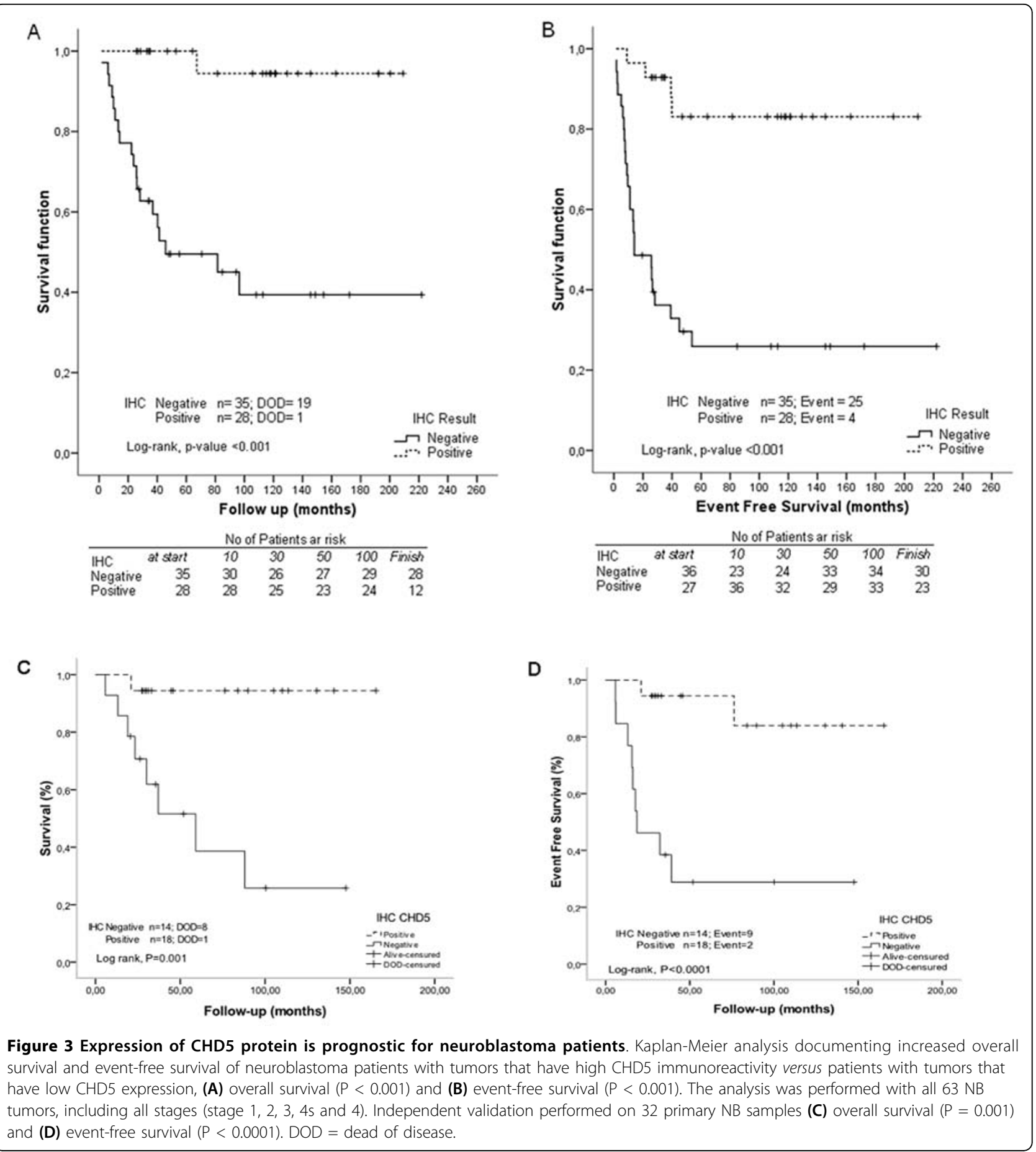

was used as a cut-off to define high $(>2)$ and low $(\leq 2)$ CHD5 expressing NB tumors. High CHD5 expression was found to be significantly associated with a better OS (log-rank test $\mathrm{P}<0.001)$ and EFS (log-rank test $\mathrm{P}<$ 0.001 ) (Figure $3 \mathrm{~A}$ and $3 \mathrm{~B}$ ). Furthermore, using this cutoff, Cox multivariate analysis showed that expression of CHD5 protein predicted OS and EFS independently of INSS stage, patient age, amplification of $M Y C N$ and $1 \mathrm{p}$
LOH (Table 2; Additional file 5). Specifically, CHD5 IHC was the only variable that remained statistically significantly associated with event-free survival in both the univariate and multivariate analyses (Table 2; Additional file 5). CHD5 IHC remained statistically significantly associated with overall survival, except when $1 \mathrm{p} \mathrm{LOH}$ was included in the multivariate analysis, owing to the strong association of CHD5 expression with 
Table 2 Cox regression analysis

\begin{tabular}{|c|c|c|c|c|c|c|}
\hline Overall Survival & & & & & & \\
\hline Variable & $\mathrm{HR}$ and $95 \% \mathrm{Cl}$ & p-value & Variable & $\mathrm{HR}$ and $95 \% \mathrm{Cl}$ & p-value & Interaction p-value ${ }^{\&}$ \\
\hline \multirow[t]{2}{*}{ CHD5 IHC } & $21.28(2.84-159.39)$ & 0.003 & & & & \\
\hline & & & CHD5 IHC & $18.67(2.27-153.88)$ & 0.007 & 0.951 \\
\hline \multirow[t]{2}{*}{ INSS } & $4.15(1.63-10.58)$ & 0.003 & INSS & $1.24(0.46-3.29)$ & 0.673 & \\
\hline & & & CHD5 IHC & $15.17(1.89-121.73)$ & 0.011 & 0.931 \\
\hline \multirow[t]{2}{*}{ Age $(>12 \mathrm{~m})$} & $5.56(1.61-19.19)$ & 0.007 & Age $(>12 \mathrm{~m})$ & $2.14(0.59-7.75)$ & 0.249 & \\
\hline & & & CHD5 IHC & $12.29(1.56-96.49)$ & 0.017 & 0.952 \\
\hline \multirow[t]{2}{*}{$\operatorname{MYCN}(*)$} & $14.27(4.28-47.58)$ & $<0.001$ & $\operatorname{MYCN}(*)$ & $8.08(2.43-26.93)$ & 0.001 & \\
\hline & & & CHD5 IHC & $14.33(1.84-111.48)$ & 0.011 & 0.946 \\
\hline $\left.\mathrm{LOH}^{* *}\right)$ & $2.88(1.04-7.96)$ & 0.042 & $\left.\mathrm{LOH}^{* *}\right)$ & $1.75(0.63-4.89)$ & 0.287 & \\
\hline \multicolumn{7}{|c|}{ Event Free Survival } \\
\hline Variable & $\mathrm{HR}$ and $95 \% \mathrm{Cl}$ & $p$-value & Variable & $\mathrm{HR}$ and $95 \% \mathrm{Cl}$ & p-value & Interaction p-value ${ }^{\&}$ \\
\hline \multirow[t]{2}{*}{ CHD5 IHC } & $8.14(2.82-23.5)$ & $<0.001$ & & & & \\
\hline & & & CHD5 IHC & 7.01 (2.09 to 23.51) & 0.002 & 0.939 \\
\hline \multirow[t]{2}{*}{ INSS } & 3.7 (1.73-7.88) & 0.001 & INSS & 1.26 (0.53 to 2.97$)$ & 0.605 & \\
\hline & & & CHD5 IHC & 7.04 (2.24 to 22.09$)$ & 0.001 & 0.638 \\
\hline \multirow[t]{2}{*}{ Age $(>12 \mathrm{~m})$} & $3.13(1.33-7.39)$ & 0.009 & Age $(>12 \mathrm{~m})$ & 1.36 (0.54 to 3.44$)$ & 0.515 & \\
\hline & & & CHD5 IHC & 5.97 (1.98 to 17.98$)$ & 0.001 & 0.933 \\
\hline \multirow[t]{2}{*}{$\operatorname{MYCN}\left({ }^{*}\right)$} & $4.58(1.84-11.42)$ & 0.001 & MYCN & 2.72 (1.07 to 6.88 ) & 0.035 & \\
\hline & & & CHD5 IHC & 6.02 (1.98 to 18.34$)$ & 0.002 & 0.492 \\
\hline $\operatorname{LOH}(* *)$ & $2.12(0.92-4.91)$ & 0.079 & LOH & 1.24 (0.52 to 2.95$)$ & 0.622 & \\
\hline
\end{tabular}

Cox regression analysis of the NB cohort using CHD5 protein expression and clinical and biologically relevant variables (INSS stage, patient age at diagnosis, MYCN amplification status and chromosome 1p LOH). Expression of CHD5 was statistically significantly associated with overall survival and event-free survival in both the univariate and multivariate analyses. All NB patients $(n=63)$ where included in the study except for the studies with MYCN amplification $(n=58)$ and $1 \mathrm{p} \mathrm{LOH}(\mathrm{n}=53)$, due to the undetermined status in some patients. IHC = Immunohistochemical analysis; INSS = International Neuroblastoma Staging System; HR $=$ hazard ratio; $\mathrm{Cl}=$ confidence interval. P-values are two sided. $\left(^{(}\right)$Interaction P-values obtained from Cox regression model: $\mathrm{IHC}+\mathrm{Co}$-factor+IHC*Co-factor. $\left({ }^{*}\right)$ data available for 58 patients; $\left.{ }^{* *}\right)$ data available for 53 patients.

chromosome 1p status (Table 2; Additional file 5). The Predictive Value of CHD5 staining was evaluated and compared to MYCN and 1p LOH (Table 3). CHD5 expression showed the highest Negative Predictive Value (NPV) for overall survival status (96.4\%) and event free survival (85.7\%). MYCN status and 1p LOH showed a NPV of $81.6 \%$ and $78.9 \%$, respectively, for the overall survival status, and of $65.3 \%$ and $63.2 \%$, respectively, for the event free survival. The Positive Predictive Value (PPV) of CHD5 expression (overall survival status: $54.3 \%$; event free survival: $71.4 \%$ ), i.e. proportion of events or deaths in patients with low CHD5 expressing tumors, was intermediate between MYCN (66.7\% and $77.8 \%$, respectively) and $1 \mathrm{p} \mathrm{LOH}$ (46.7\% and $60 \%$, respectively) values. CHD5 IHC showed high sensitivity and accuracy rate for the prediction of OS (95\% and 73\%, respectively) and EFS (86.20\% and $77.80 \%)$ (Table 4).

The prognostic value of CHD5 expression was validated on an independent, blinded set of 32 FFPE primary NB tumors of patients diagnosed and treated at the Children's Hospital of Boston $(n=21)$ and HSJD of

Table 3 Analysis of the Predictive Value was performed for a descriptive comparison between CHD5 expression and MYCN and 1p LOH

\begin{tabular}{|c|c|c|c|c|c|c|c|c|c|}
\hline & & \multicolumn{4}{|c|}{ Overall Survival } & \multicolumn{4}{|c|}{ Event Free Survival } \\
\hline & & Alive & Dead & PPV & NPV & No event & Event & PPV & NPV \\
\hline \multirow[t]{2}{*}{ CHD5 IHC } & High & $27(62.8 \%)$ & $1(5 \%)$ & $54.30 \%$ & $96.40 \%$ & $24(70.6 \%)$ & $4(13.8 \%)$ & $71.40 \%$ & $85.70 \%$ \\
\hline & Low & $16(37.2 \%)$ & 19 (95\%) & & & $10(29.4 \%)$ & $25(86.2 \%)$ & & \\
\hline \multirow[t]{2}{*}{ MYCN } & Non amplified & $40(93 \%)$ & $9(60 \%)$ & $66.70 \%$ & $81.60 \%$ & $32(94.1 \%)$ & $17(70.8 \%)$ & $77.80 \%$ & $65.30 \%$ \\
\hline & Amplified & $3(7 \%)$ & $6(40 \%)$ & & & $2(5.9 \%)$ & $7(29.2 \%)$ & & \\
\hline \multirow[t]{2}{*}{$1 \mathrm{p} \mathrm{LOH}$} & No & $30(78.9 \%)$ & $8(53.3 \%)$ & $46.70 \%$ & $78.90 \%$ & $24(80 \%)$ & $14(60.9 \%)$ & $60 \%$ & $63.20 \%$ \\
\hline & LOH & $8(21.1 \%)$ & $7(46.7 \%)$ & & & $6(20 \%)$ & $9(39.1 \%)$ & & \\
\hline
\end{tabular}


Table 4 Comparison of sensitivity, specificity and accuracy rate between CHD5 expression, MYCN status and 1p LOH

\begin{tabular}{ccccccc}
\hline & \multicolumn{3}{c}{ Overall Survival } & \multicolumn{3}{c}{ Event Free Survival } \\
\cline { 2 - 7 } & Sens & Specif & Accurancy & Sens & Specif & Accurancy \\
\hline CHD5 IHC & $95.00 \%$ & $62.80 \%$ & $73.00 \%$ & $86.20 \%$ & $70.60 \%$ & $77.80 \%$ \\
\hline MYCN & $40.00 \%$ & $93.00 \%$ & $79.30 \%$ & $29.20 \%$ & $94.10 \%$ & $67.20 \%$ \\
\hline 1p LOH & $46.70 \%$ & $78.90 \%$ & $69.80 \%$ & $39.10 \%$ & $80.00 \%$ & $62.30 \%$ \\
\hline
\end{tabular}

Barcelona $(\mathrm{n}=11)$. Kaplan-Meier analysis and a logrank test showed a statistically significant difference in OS (log-rank test $\mathrm{P}=0.001)$ and EFS (log-rank test $\mathrm{P}<$ $0.0001)$ between patients with high and low CHD5 expression scores (Figure $3 \mathrm{C}$ and $3 \mathrm{D}$ ). Tumors with high IHC scores were associated with longer survival (mean 73 months) in comparison with low expressing tumors (mean 46 months).

These results suggest that $\mathrm{CHD} 5$ protein expression is a potential prognostic marker of outcome in $\mathrm{NB}$ patients.

\section{CHD5 expression reactivation is associated with tumor response to induction therapy}

Tumor histology and gene expression can change with treatment as a result of important changes in cellular processes. We investigated the effects of induction chemotherapy (3 cycles) on CHD5 expression in 12 high risk NB cases with available paired diagnostic and postchemotherapy tumor specimens for qRT-PCR and immunohistochemical analyses. At diagnosis all these tumors (2 locoregional and 10 stage $4 \mathrm{NB}$ ) displayed low CHD5 mRNA expression and negative immunostaining. Following induction chemotherapy, a significant increase of $\mathrm{CHD} 5$ transcript and CHD5 positive nuclear staining was detected in $6 / 12$ specimens, together with therapy-induced morphological changes (increased cytoplasm and ganglion-like cell morphology) (Figure 4A and 4C; cases \#1-6). All these patients achieved an initial complete or very good response to cytotoxic therapy (chemo- and radiation therapy). At the time of analysis, $5 / 6$ patients were alive with a mean follow-up of 35.62 months (Figure 4B). One case, stage $4 M Y C N$ amplified, progressed after a good initial response to chemotherapy and died of refractory bone marrow disease (Figure 4C; case \#6). Bone marrow aspirate smears of this patient exhibited widespread tumor dissemination with CHD5 negative neuroblast aggregates (data not shown).

In contrast, low gene and protein expression levels persisted in the 6 remaining post-therapy specimens $(6$ stage $4 \mathrm{NB}$; $3 / 6$ MYCN amplified and $1 \mathrm{p} 36$ deleted tumors) (Figure 4A and 4C; cases \#7-12). Therapy induced neuroblastic differentiation was observed in only one of these samples (case \#7), a stage 4 NB with aberrant morphological changes. All 6 patients died of rapid disease progression with no signs of clinical response; with a mean survival of 12.73 months (Figure $4 B)$.

These observations suggest a relationship between CHD5 expression reactivation and response to induction therapy and subsequent patient outcome.

\section{Discussion}

Gene expression of CHD5, an ATP-dependent chromatin remodeling enzyme, has been reported to be restricted essentially to the nervous system $[8,10]$. We describe for the first time that CHD5 is a neuron specific protein in normal neural tissue, with variable immunostaining intensity and intracellular localization among the neuron types of the cerebral cortex. Recent evidences suggest that the diverse neuron cell classes derive from distinct embryonal germinal zones and are characterized by specific cell signaling systems that regulate neural stem cells throughout the developing brain [13-15]. Thus, neuronal cells adopt a brain layer fate determined by their molecular profiles [14]. While we did not observe a layer specific distribution of CHD5 in the cerebral cortex, we did note an association of CHD5 expression with neurons with distinct morphological, physiological and neurochemical features.

In normal neural tissue, glial cells appeared consistently devoid of CHD5 expression. In human glial tumors, chromosome arm $1 \mathrm{p}$ allelic loss is a frequent genetic abnormality, especially in oligodendrogliomas (70-85\%) and astrocytomas (20-30\%) [16]. Recently, low levels of CHD5 expression have been reported in gliomas with $1 p$ deletion, whereas nondeleted tumors displayed expression levels comparable to normal brain [4]. Thus, deletion of CHD5 has been proposed as an initiating event in gliomas [4]. Our findings, however, suggest that the role of CHD5 as a tumor suppressor in glial tumors needs further investigation.

NTs are embryonal cancers that are assumed to originate from primitive sympathetic neuroblast aggregates located in neural crest derived sympathetic nervous system. We observed how primitive neuroblast aggregates found in fetal adrenal gland specimens generally lack CHD5 expression. Interestingly, only a few cells were found with a variable degree of nuclear reactivity in larger aggregates. To date, the fate of these immature neuroblastic aggregates remains unsolved, and spontaneous involution and cell maturation have been proposed [17]. 




The immunoreactivity observed in a small proportion of neuroblasts within these islets could suggest the establishment of CHD5 expression prior to their disappearance; however, no evident differentiating features were observed in these immunopositive cells that suggested the activation of the maturation process.

In NTs, CHD5 is essentially expressed in the nucleus of differentiating neuroblastic cells and ganglion cells, and absent in the Schwannian stromal component. However, the most intense immunoreactivity was observed in stage $4 \mathrm{~s} \mathrm{NB}$, a rare subgroup of histologically undifferentiated, highly proliferative, metastatic tumors with a high incidence of spontaneous regression, affecting young infants. Accurate distinction of spontaneously regressing infant NB from high risk infant stage 4 can be difficult, but critical for therapeutic decisions. In our hands, the intensely positive CHD5 nuclear staining enabled a clear distinction of stage $4 \mathrm{~s}$ NB from stage $4 \mathrm{NB}$, which was consistently immunonegative. These results are consistent with our previous gene expression profiling study, where similar differential CHD5 expression profiles were observed amongst infants with disseminated NB subgroups [18]. Thus, CHD5 immunohistochemical staining may be clinically useful for a more accurate characterization of disseminated infant NB.

In NB, CHD5 nuclear staining was strongly associated with established favorable prognostic variables 
like low clinical stage, age at diagnosis $<12$ months and favorable histology. Our findings suggest that CHD5 protein expression may accurately define NB risk groups and may, therefore, be a prognostic marker. Evidence is provided by the statistically significant association found between high CHD5 immunoreactivity and favorable OS and EFS. These results are consistent with recent studies reporting a strong association of CHD5 mRNA levels with patient outcome in NB $[5,10]$. Furthermore, Cox multivariate analyses suggest that the prognostic value of CHD5 protein expression is independent of other clinical and biological variables currently used in risk stratification of NB patients and could therefore represent an immunohistochemical marker of prognosis in NB.

Currently, risk stratification of NB patients is performed by combining different markers with strong prognostic impact, including patients' age at diagnosis, tumor stage, genomic amplification of the oncogene $M Y C N$, copy number alterations of chromosomal regions $1 \mathrm{p}, 11 \mathrm{q}$ and $17 \mathrm{q}$, tumor DNA content $[1,19]$ and Shimada histological score [20]. However, despite elaborate risk stratification strategies, outcome prediction in neuroblastoma is still deficient. In recent years, to improve risk assessment additional prognostic indicators such as gene-expression signatures [21-23], combined genomic and molecular signatures [24] or expression levels of single candidate genes, e.g., Trk (NTRK) family of neurotrophin receptors [25,26], FYN [27], PRAME [28] and ZNF423 [29], have been associated with NB clinical behavior. Expression of the Trk family receptors has been the most extensively characterized marker in NB and has been found to be consistently correlated with the biology and clinical behavior of NB. Based on our results, there is an apparent similarity between the expression patterns of CHD5 and TRKA in NB and their patterns of association with NB disease outcome. TRKA expression has been reported to be high in biologically favorable NB tumors and inversely associated with MYCN amplification [30]. The prognostic value of the immunohistochemical detection of TrkA has also been examined and reported to be high, especially in combination with Ha-Ras expression pattern $[31,32]$. Further IHC studies have correlated the lack of TrkA expression with metastatic malignant NB [33]. However, in the latter study, $34 \%$ of the patients with stage 4 NB displayed TrkA expression, a subset of which died of aggressive metastatic disease despite TrkA expression [33,34]. In our study, the majority of stage 4 NB either lacked CHD5 immunoreactivity (83\%) or exhibited weak nuclear staining (13\%), a high risk phenotype according to our scoring system. Only one stage 4 tumor was found to be clearly immunoreactive for CHD5; at the time of analysis the patient is alive, 29 months from diagnosis. These observations further confirm CHD5 as a powerful prognostic marker that could complement other known markers such as age at diagnosis, stage, MYCN status, cellular DNA content, 1p deletion and tumor histology. However, the potential clinical use of this marker must be tested in larger, prospective cohorts.

It is known that tumor histology and gene expression can change with treatment as a result of important changes in cellular processes, e.g., induced tumor differentiation, DNA repair, apoptosis and tissue necrosis. Undifferentiated NB occasionally exhibit neuroblastic maturation in response to chemotherapy. Assessment of CHD5 gene and protein expression in NB post-therapy specimens revealed that tumors with evident neuroblastic maturation showed both CHD5 gene and protein reactivation. Notably, none of these tumors harbored $1 \mathrm{p}$ deletion. Conversely, in tumors where minimal or no morphological changes were observed in the post-treatment specimens, low CHD5 expression persisted. These observations suggest the existence of a subset of tumors within high risk NB where CHD5 expression can be reactivated from the silenced state by standard chemotherapy. Remarkably, when posttherapy reactivation was observed, $C H D 5$ expression was largely associated with disease response to cytotoxic induction therapy and subsequently with longer patient OS. All 12 patients included in the study received the same treatment, nevertheless some tumors failed to respond. At present, treatment response in NB is routinely evaluated by monitoring urine levels of catecholamine and its metabolites (VMA/HVA ratio) and by estimating the decrease in the size of measurable lesions with conventional imaging modalities, such as computed tomography (CT) or magnetic resonance imaging (MRI). At the time of second-look surgery, the degree of induced tumor cell differentiation and the extent of necrosis can also be useful to estimate treatment response. However, no biological markers for tumor chemotherapy responsiveness have been reported in NB. The use of such biomarkers would make chemotherapy more effective for individual patients by allowing timely changes of therapy in the case of nonresponding tumors. Furthermore, markers reflecting tumor response can function as surrogates of long-term outcome. Taking into account the small cohort of cases that may have led to an overestimation of the data, our findings would suggest that restoration of CHD5 expression could be a surrogate marker of treatment response that can be clinically useful to identify patients that do not benefit from conventional treatment. These results warrant further investigation in a larger cohort of uniformly treated patients.

In summary, we report that the differential expression of the neuron-specific protein CHD5 accurately defines NB risk groups and may represent a marker of outcome in neuroblastoma that can be tested by conventional 
immunohistochemistry. In high risk NB patients, reestablishment of CHD5 expression following chemotherapy should be tested prospectively as a surrogate marker of treatment response.

\section{Additional material}

Additonal file 1: Clinical and biological characteristics of 90 NT specimens included in the study. INSS = International Neuroblastoma Staging System; Diagnosis: NB = neuroblastoma, GNB =

ganglioneuroblastoma, $\mathrm{GN}=$ ganglioneuroma; Disease status: $\mathrm{A}=$ alive, $\mathrm{D}=$ dead; $\mathrm{EFS}=$ Event free survival; $\mathrm{QRT}-\mathrm{PCR}=$ Quantitative real-time $P C R ; I H C=$ immunohistochemistry; $\mathrm{n} . \mathrm{a}=$ not available data Immunohistochemical analysis of CHD5 expression in NTs Results are displayed as percentage of CHD5 immunopositive cells present in each tumor specimen. Staining intensity: $0=$ negative; $1=$ weak staining: $2=$ strong staining, $3=$ very intense staining. Proportion of positive cells values $(<25 \%=1 ; 25-75=2 ;>75 \%=3)$. Intensity and positive cell values were multiplied together to provide a single score for each case.

Additonal file 2: A. Immunohistochemical staining of FFPE sections of two immunopositive neuroblastic tumors using two different batches of the anti-CHD5 antibody (T00251-A1 and T00251-A02); B. Immunochemical assay with the anti-CHD5 antibody (Strategic Diagnostics, DE) on mouse xenografts derived from human NB cell lines. The specificity of the anti-CHD5 antibody was validated by immunohistochemical assays on FFPE sections of mouse xenografts of human NB cell lines (SK-N-JD, SK-N-LP and SK-N-AS). In these NB cell lines CHD5 gene expression is very low or absent (data not shown), similar to previously reported data (ref. 5 , ref. 10). Two different antiCHD5 antibody batches (T00251-A1 and T00251-A02, Strategic Diagnostics, DE) were tested. Ganglioneuroblastoma FFPE tissue sections were used as positive control samples. All the analyzed xenographs were composed nearly exclusively (>95\%) of neuroblastic cells exhibiting no CHD5 nuclear staining and faint cytoplasmic staining (when present). Only few $(<5 \%)$ immunopositve cells were observed in the SK-N-LP xenograft. However, viable tumor cells in the SK-N-LP xenograft where negative for CHD5 nuclear staining, similar to SK-N-JD and SK-N-AS. These results were comparable to the immunostaining pattern observed in undifferentiated high risk NB tumors. The GNB ganglionar cells showed intense nuclear and diffused cytoplasm immunostaining.

Additional file 3: CHD5 mRNA expression levels in NTs Results are displayed as mean expression levels of NT subgroups obtained from two independent analyses. $\mathrm{HR}=$ high risk NB (Stage 4 and Stage 3 MYCN amplified); $L R=$ low risk NB (stage 1, stage 2 and stage 3 MYCN nonamplified); $\mathrm{GNB}=$ ganglioneuroblastoma; $\mathrm{GN}$ = ganglioneuroma. Quantification was performed relative to normal fetal brain. Error bars illustrate the variability amongst the samples of each NT subgroup.

\section{Additional file 4: Comparison of CHD5 mRNA and protein} expression. The lineal graph shows comparison between CHD5 mRNA levels and protein immunoreactivity in $34 \mathrm{NB}$ cases. Low CHD5 protein scores were associated with lower mRNA levels (negative $z$-score values), and high IHC scores with high mRNA expression (positive $z$-score values), (Spearman's correlation analysis rho $=0.774 ; \mathrm{P}<0.001$ ). Low risk tumors, stage $4 \mathrm{~s}$ and infant stage $1 \mathrm{NB}$ tumors showed very intense nuclear staining in comparison to the observed transcript levels (cases \# 1-6, 30, 31 and 33).

Additional file 5: Cox multivariate análisis. Cox multivariate regression analysis has been performed using clinical and biological variables currently used in risk stratification of NB patients (INSS stage, age at diagnosis, MYCN status and $1 \mathrm{p} \mathrm{LOH}$ ) in combination with the CHD5 IHC. The analysis has been performed sequentially, adding one variable at each step, in order to assess how the presence of each variable influences the performance of CHD5. CHD5 IHC remained statistically significantly associated with overall survival in all the analyses, except when the $1 \mathrm{p} \mathrm{LOH}$ parameter is included in the overall survival analysis. This is due to the strong association of the expression of CHD5, located on $1 p 36$, with chromosome $1 p$ status. All the rest of variables, except for
MYCN amplification, were not statistically significant. For event free survival analysis, CHD5 IHC is the only variable that remained statistically significant along the whole analysis, even in the presence of $1 \mathrm{p} \mathrm{LOH}$. IHC $=$ Immunohistochemical analysis; INSS = International Neuroblastoma Staging System; HR = hazard ratio; $\mathrm{Cl}=$ confidence interval. P-values are two sided.

\section{Acknowledgements}

Authors thank Dr. B. Spengler (Fordham University, New York) and Dr. N.K.V. Cheung (MSKCC, New York) for annotated NB cell lines; the Neural Tissue Bank (Hospital Clínic, Barcelona) for normal brain samples and the Department of Audiovisual Systems (HSJD, Barcelona) for technical assistance. This study was supported by grants from the Spanish Ministry of Health (PI070286), Spanish Society against Cancer (Asociación Española Contra el Cáncer, 2007), the Catalan government (2005SGR00605; 2006Fl00404), and the generous donations from Margarita del Pozo and Alicia Pueyo Foundations.

\section{Author details}

'Developmental Tumor Biology Laboratory, Hospital Sant Joan de Déu, Fundación Sant Joan de Déu, Barcelona, Spain. ²Department of Pathology, Hospital Sant Joan de Déu, Barcelona, Spain. ${ }^{3}$ Department of Neurology, University of North Carolina, Chapel Hill, USA. ${ }^{4}$ Laboratory of Biostatistics \& Epidemiology, Universitat Autònoma de Barcelona; Clinical Pharmacology Service, IDIBAPS, Hospital Clinic, Barcelona, Spain. ${ }^{5}$ Department of Pediatrics, Memorial Sloan-Kettering Cancer Centre, New York, USA. ${ }^{6}$ Division of Pediatric Hematology/Oncology, Dana-Farber Cancer Institute and Children's Hospital of Boston, USA. 'Department of Pathology, Children's Hospital of Boston, USA.

\section{Authors' contributions}

$\mathrm{CL}, \mathrm{IG}$ and $\mathrm{JM}$ are responsible for the initial conception and overall hypothesis of this study. IG, GM and $\mathrm{CL}$ are responsible for the design of this manuscript, including the original draft and subsequent revisions. IG, GM, ER, MS, TG, JR, NKC, CdT, JM MK, RG, AAP and CL were involved with the interpretation of data, draft and revision of this manuscript. CdT provided guidance for many of the experiments. NKC, TG, MK, RG and AAP are responsible for the procurement and cryopreservation of NBT tissue specimens derived from MSKCC and CHB/DFCI. ER, IG, GM, JM and CL were responsible for the procurement and cryopreservation of NBT tissue specimens derived from the Spanish institutions. ER, IG GM, CL and MS are responsible of inmunohistochemical analyses. MS evaluated tumour specimens for staging classification, tumour content. JM and $\mathrm{CL}$ are responsible for patient clinico-biological database management. CC, GM, PG and $E R$ are responsible for the in vivo study. IG, GM and $C L$ are responsible for the quantitative $P C R$ experiments and inmunoblotting. JR and $C L$ are responsible of statistical analyses. All authors were involved in the drafting and revisions for this manuscript. All authors read and approved the final manuscript.

\section{Competing interests}

The authors declare that they have no competing interests.

Received: 25 January 2010 Accepted: 15 October 2010 Published: 15 October 2010

\section{References}

1. Maris JM, Hogarty MD, Bagatell R, Cohn SL: Neuroblastoma. Lancet 2007, 369:2106-2120.

2. Weith A, Brodeur GM, Bruns GA, Matise TC, Mischke D, Nizetic D, Seldin MF, van Roy N, Vance J: Report of the second international workshop on human chromosome 1 mapping 1995. Cytogenet Cell Genet 1996, 72:114-144.

3. Fong CT, Dracopoli NC, White PS, Merrill PT, Griffith RC, Housman DE, Brodeur GM: Loss of heterozygosity for the short arm of chromosome 1 in human neuroblastoma: correlation with $\mathrm{N}$-myc amplification. Proc Natl Acad Sci USA 1989, 86:3753-3757. 
4. Bagchi A, Papazoglu C, Wu Y, Capurso D, Brodt M, Francis D, Bredel M, Vogel H, Mills AA: CHD5 is a tumor suppressor at human 1p36. Cell 2007, 128:459-475.

5. Fujita T, Igarashi J, Okawa ER, Gotoh T, Manne J, Kolla V, Kim J, Zhao H, Pawel BR, London WB, Maris JM, White PS, Brodeur GM: CHD5, a tumor suppressor gene deleted from $1 \mathrm{p} 36.31$ in neuroblastomas. J Nat/ Cancer Inst 2008, 100:940-949.

6. Okawa ER, Gotoh T, Manne J, Igarashi J, Fujita T, Silverman KA, Xhao H, Mosse YP, White PS, Brodeur GM: Expression and sequence analysis of candidates for the $1 \mathrm{p} 36.31$ tumor suppressor gene deleted in neuroblastomas. Oncogene 2008, 27:803-810.

7. Mulero-Navarro S, Esteller M: Chromatin remodeling factor CHD5 is silenced by promoter $\mathrm{CpG}$ island hypermethylation in human cancer. Epigenetics 2008, 3:210-215.

8. Marfella CG, Imbalzano AN: The Chd family of chromatin remodelers. Mutat Res 2007, 618:30-40.

9. Schuster EF, Stöger R: CHD5 defines a new subfamily of chromodomainSWI2/SNF2-like helicases. Mammalian Genome 2002, 13:117-119.

10. Thompson PM, Gotoh T, Kok M, White PS, Brodeur GM: CHD5, a new member of the chromodomain gene family, is preferentially expressed in the nervous system. Oncogene 2003, 22:1002-1011.

11. Brodeur GM, Pritchard J, Berthold F, Carlsen NL, Castel V, Castelberry RP, De Bernardi B, Evans AE, Favrot M, Hedborg F: Revision of the International criteria for neuroblastoma diagnosis, staging and response to treatment. J Clin Oncol 1993, 11:1466-1477.

12. Lavarino C, Garcia I, Mackintosh C, Cheung NKV, Domenech G, Ríos J, Perez N, Rodríguez E, De Torres C, Gerald WL, Tuset E, Acosta S, Beleta H, de Alava E, Mora J: Differential expression of genes mapping to recurrently abnormal chromosomal regions characterize neuroblastic tumours with distinct ploidy status. BMC Med Genomics 2008, 1:36

13. Anderson SA, Kaznowski CE, Horn C, Rubenstein JL, McConnell SK: Distinct origins of neocortical projection neurons and interneurons in vivo. Cereb Cortex 2002, 12:702-709.

14. Hevner RF, Daza RA, Rubenstein JL, Stunnenberg H, Olavarria JF, Englund C: Beyond laminar fate: toward a molecular classification of cortical projection/pyramidal neurons. Dev Neurosci 2003, 25:139-151.

15. Gilbertson RJ, Ellison DW: The origins of medulloblastoma subtypes. Annu Rev Pathol 2008, 3:341-365.

16. Barbashina V, Salazar P, Holland EC, Rosenblum MK, Ladanyi M: Allelic losses at 1 p36 and 19q13 in gliomas: correlation with histologic classification, definition of a 150-kb minimal deleted region on $1 \mathrm{p} 36$, and evaluation of CAMTA1 as a candidate tumor suppressor gene. Clin Cancer Res 2005, 11:1119-1128.

17. de Preter K, Vandesompele J, Heimann P, Yigit N, Beckman S, Schramm A, Eggert A, Stallings RL, Benoit Y, Renard M, De Paepe A, Laureys G, Påhlman S, Speleman F: Human fetal neuroblast and neuroblastoma transcriptome analysis confirms neuroblast origin and highlights neuroblastoma candidate genes. Genome Biol 2006, 7(9):R84

18. Lavarino C, Cheung NK, Garcia I, Domenech G, de Torres C, Alaminos M, Rios J, Gerald WL, Kushner B, LaQuaglia M, Mora J: Specific gene expression profiles and chromosomal abnormalities are associated with infant disseminated neuroblastoma. BMC Cancer 2009, 3:9-44.

19. Ambros PF, Ambros IM, Brodeur GM, Haber M, Khan J, Nakagawara A, Schleiermacher G, Speleman F, Spitz R, London WB, Cohn SL, Pearson AD, Maris JM: International consensus for neuroblastoma molecular diagnostics: report from the International Neuroblastoma Risk Group (INRG) Biology Committee. Br J Cancer 2009, 100:1471-1482.

20. Shimada H, Ambros IM, Dehner LP, Hata J, Joshi W, Roald B, Stram DO, Gerbing RB, Lukens JN, Matthay KK, Castleberry RP: The International Neuroblastoma Pathology Classification (the Shimada system). Cancer 1999, 86:364-72

21. Oberthuer A, Berthold F, Warnat P, Hero B, Kahlert Y, Spitz R, Ernestus K, König R, Haas S, Eils R, Schwab M, Brors B, Westermann F, Fischer M: Customized oligonucleotide microarray gene expression-based classification of neuroblastoma patients outperforms current clinical risk stratification. J Clin Oncol 2006, 24(31):5070-5078.

22. De Preter $K$, Vermeulen J, Brors B, Delattre O, Eggert A, Fischer $M$, JanoueixLerosey I, Lavarino C, Maris JM, Mora J, Nakagawara A, Oberthuer A, Ohira M, Schleiermacher G, Schramm A, Schulte JH, Wang Q, Westermann F, Spleleman F, Vandesompele J: Accurate outcome prediction in neuroblastoma across independent data sets using a multigene signature. Clin Cancer Res 2010, 16(5):1532-1541.

23. Oberthuer A, Hero B, Berthold F, Juraeva D, Faldum A, Kahlert $Y$, Asgharzadeh S, Seeger R, Scaruffi P, Tonini GP, Janoueix-Lerosey I, Delattre O, Schleiermacher G, Vandesompele J, Vermeulen J, Speleman F, Noguera R, Piqueras M, Bénard J, Valent A, Avigad S, Yaniv I, Weber A, Christiansen H, Grundy RG, Schardt K, Schwab M, Eils R, Warnat P, Kaderali L, Simon T, Decarolis B, Theissen J, Westermann F, Brors B, Fischer M: Prognostic impact of gene expression-based classification of neuroblastoma. J Clin Oncol 2010, 28(21):3506-15.

24. Tomioka N, Oba S, Ohira M, Misra A, Fridlyand J, Ishii S, Nakamura Y, Isogai E, Hirata T, Yoshida Y, Todo S, Kaneko Y, Albertson DG, Pinkel D, Feuerstein $B G$, Nakagawara A: Novel risk stratification of patients with neuroblastoma by genomic signature, which is independent of molecular signature. Oncogene 2008, 27:441-449.

25. Nakagawara A, Arima-Nakagawara M, Scavarda NJ, Azar CG, Cantor AB, Brodeur GM: Association between high levels of expression of the TRK gene and favorable outcome in humana neuroblastoma. $N$ Engl J Med 1993, 328:847-854

26. Brodeur GM, Minturn JE, Ho R, Simpson AM, lyer $R$, Varela $C R$, Light JE, Kolla V, Evans AE: Trk receptor and inhibition in neuroblastomas. Clin Can Res 2009, 15(10):3244-3250.

27. Berwanger B, Hartmann O, Bergmann E, Bernard S, Nielsen D, Krause M, Kartal A, Flynn D, Wiedemeyer R, Schwab M, Schäfer H, Christiansen H, Eilers M: Loss of a FYN-regulated differentiation and growth arrest pathway in advanced stage neuroblastomas. Cancer Cell 2002, 2(5):377-86.

28. Oberthuer A, Hero B, Spitz R, Berthold F, Fischer M: The tumor-associated antigen PRAME is universally expressed in high-stage neuroblastoma and associated with poor outcome. Clin Can Res 2004, 10(13):4307-13.

29. Huang S, Laoukili J, Epping MT, Koster J, Hölzel M, Westerman BA, Nijkamp W, Hata A, Asgharzadeh S, Seeger RC, Versteg R, Beijersbergen RL, Bernards R: ZNF423 is critically required for retinoic acid-induced differentiation and is a marker of neuroblastomas outcome. Cancer Cell 2009, 15:328-340.

30. Nakagawara A, Arima M, Azar CG, Scavarda NJ, Brodeur GM: Inverse relationship between trk expression and $\mathrm{N}$-myc amplification in humana neuroblastomas. Cancer Res 1992, 52:1364-1368.

31. Tanaka $T$, Hiyama E, Sugimoto $T$, Sawada $T$, Tanabe $M$, Ida N: trkA gene expression in neuroblastoma. Can Res 1995, 76:1086-1095.

32. Tanaka T, Sugimoto T, Sawada T: Prognostic discrimination among neuroblastomas according to Ha-ras/trk A gene expression. Can Res 1998, 83:1626-1633.

33. Krammer K. Gerald W, LeSauteur L, Saragovi HU, Cheung N-KV: Prognostic value of TrkA protein detection by monoclonal antibody $5 \mathrm{C} 3$ in neuroblastoma. Clin Can Res 1996, 2:1361-67.

34. Krammer K. Gerald W, LeSauteur L, Saragovi HU, Cheung N-KV: Monoclonal antibody to human Trk-A: Diagnostic and therapeutic potential in neuroblastoma. Eur J Cancer 33(12):2090-2091.

doi:10.1186/1476-4598-9-277

Cite this article as: Garcia et al:: Expression of the neuron-specific protein CHD5 is an independent marker of outcome in neuroblastoma. Molecular Cancer 2010 9:277.

\section{Submit your next manuscript to BioMed Central and take full advantage of:}

- Convenient online submission

- Thorough peer review

- No space constraints or color figure charges

- Immediate publication on acceptance

- Inclusion in PubMed, CAS, Scopus and Google Scholar

- Research which is freely available for redistribution

Submit your manuscript at www.biomedcentral.com/submit
C Biomed Central 